\title{
КВАНТОВО-ЭЛЕКТРОДИНАМИЧЕСКАЯ МОДЕЛЬ НОРМАЛЬНЫХ МЕТАЛЛОВ
}

\author{
(Представил В. Хижняков)
}

Метод расчета электронной структуры твердого тела, предложенный в $\left[{ }^{1,2}\right]$ и использованный для вычисления энергетических электронных спектров лития с объемноцентрированной кубической (ОЦК) структурой и бериллия с гексагональной плотноупакованной структурой $\left[{ }^{3}\right]$, оказался несколько трудоемким при расчете электронной структуры болеє сложных твердых тел. В целях уменьшения объема вычислительных работ мы предлагаем здесь новый метод, который развивает идеи метода $\left[{ }^{1,2}\right]$ и представляет собой попытку совершенствования квантовой теории свободных электронов в твердом теле.

\section{1. Введение}

Изложим идею нового метода, в котором, как и в $\left[{ }^{2}\right]$, твердое тело рассматривается как система, состоящая из электронного и ядєрного полей, взаимодействующих через электромагнитное поле. Для этого введем унитарный оператор, трансформирующий векторы состояний и операторы энергии в новое представление, в котором хотя бы один внешний электрон твердого тела описывается плоской волной $K_{\text {кs }}=V^{-1 / 2} \exp i k x C_{s}$, где $C_{s}-$ спиновая функция, $V-$ объем твердого тела, $\boldsymbol{k}$ - квазиимпульс электрона, $s-$ спин электрона. При этом одноэлектронные функции остальных внешних электронов незначительно отличаются от плоских волн. Поэтому в новом представлении мы переходим к приближению, в котором все внешние электроны описываются плоскими волнами. Приближенные одноэлектронные функции внутренних электронов в новом представлении $\widetilde{B}_{\mathrm{ks}}^{(r)}$ строим из таковых функций в старом представлении $B_{\mathrm{ks}}^{(r)}$ путем ортогонализации последних к плоским волнам и определения входящих в них параметров из вариационного принципа. Имеем

$$
\begin{gathered}
\widetilde{B}_{\mathrm{Ks}}^{(r)}=B_{\mathrm{Ks}}^{(r)}-\sum_{\mathrm{K}^{\prime} s^{\prime}}\left\langle K_{\mathrm{K}^{\prime} s^{\prime}} \mid B_{\mathrm{Ks}}^{(r)}\right\rangle K_{\mathrm{K}^{\prime} s^{\prime}}-\sum_{l=r+1}^{\tilde{n}-1} \sum_{\mathrm{K}^{\prime} s^{\prime}}\left\langle\widetilde{B}_{\mathrm{K}^{\prime} s^{\prime}}^{(l)} \mid B_{\mathrm{Ks}}^{(r)}\right\rangle \cdot \widetilde{B}_{\mathrm{K}^{\prime} s^{\prime}}^{(l)} \\
(r=1, \ldots, \tilde{n}-1),
\end{gathered}
$$

где $\tilde{n}-1-$ число подмножеств внутренних электронов,

$$
B_{\mathrm{K} s}^{(r)}=C_{\mathrm{Kr} r} N^{-1 / 2} \sum_{1} \exp (-i k l) \cdot \varphi_{r}(x-l) C_{s}
$$

- волновая функция Блоха внутреннего электрона $r$-й группы типа ЛКАО в старом представлении,

$$
\varphi_{r}(x-l)=D_{r} \Pi_{r}(x) \exp \left(-\omega_{r}|x|\right),
$$

$D_{r}$ - нормировочный коэффициент функции $\quad \varphi_{r}(x), A_{\mathrm{kr}}=C_{\mathrm{k} r} \cdot D_{r}-$ нормировочный коэффициент (обеспечивающий нормировку функ- 
ции $\left.\tilde{B}_{\mathrm{\kappa} s}^{(r)}\right), \Pi_{r}(x)$ - полином от $\boldsymbol{x}, \omega_{r}=\omega_{0 r} / a_{0}-$ вариационный параметр, $N_{y}$ - число узлов в решетке, $\boldsymbol{l}$ - радиус-вектор узла решетки.

Одноэлектронное уравнение в новом представлении, собственными функциями которого являются функции $\left\{K_{\text {кs }}\right\}$ и $\left\{\widetilde{B}_{\mathrm{ks}}^{(r)}\right\}$, содержит потенциальную энергию

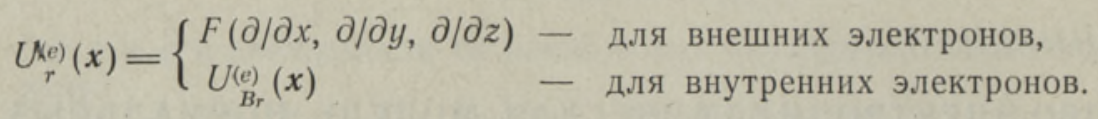

Используя оператор энергии этого уравнения, строим невозмущенный квантово-полевой оператор энергии $\hat{H}_{0}$ электронного поля. При этом квантово-полевой оператор энергии возмущения $\hat{H}_{i}$ равен разности между заданным квантово-полевым оператором энергии $\hat{H}$ электронного поля (записанным с учетом его взаимодействия с электромагнитным полем) и оператором энергии $\hat{H}_{0}$. Энергетический электронный спектр твердого тела определяется по формуле (22) из [ $\left.{ }^{4}\right]$ (или по (1.27) из [5]).

Мы вводим следующие приближения: 1) полагаем, что во втором члене правой части (1) волновой вектор $\boldsymbol{k}^{\prime}$ принадлежит области $k$-пространства, занятой внешними электронами, и усредняем $\left\langle\boldsymbol{k}^{\prime 2}\right\rangle$ по этой области; 2) пренебрегаем в (1) членами, пропорциональными второй и третьей степени выражения $\left\langle K_{\kappa^{\prime} s^{\prime}} \mid B_{\mathrm{ks}}^{(r)}\right\rangle$, что эквивалентно замене (1) функцией

$$
\widetilde{B}_{\mathrm{K} s}^{(r)} \approx B_{\mathrm{Ks}}^{(r)}-\sum_{\mathrm{K}^{\prime} s^{\prime}}\left\langle K_{\mathrm{K}^{\prime} s^{\prime}} \mid B_{\mathrm{Ks}}^{(r)}\right\rangle K_{\mathrm{K}^{\prime} s^{\prime}}
$$

3) полагаем, что атомные волновые функции остовных электронов, взятые для двух разных узлов решетки, приближенно ортогональны; 4) потенциальную энергию $U_{B r}^{(e)}$, входящую в формулу (4), аппроксимируем следующим выражением:

где

$$
\underset{B_{r}}{U(e)}=-\mathrm{e}_{0}^{2}\left(Z-\delta_{r}\right) L\left(c_{r}, d_{r}\right),
$$

$$
L\left(c_{r}, d_{r}\right)=\sum_{1}|x-l|^{-1}-\sum_{1 \neq 0} c_{r}|l|^{-1}-d_{r} V_{0}^{-1} \int d^{3} x|x|^{-1},
$$

$\delta_{r}$ - некоторый параметр, $e_{0} Z$ - заряд узла решетки (ядра), $\mathrm{e}_{0}\left(Z-\delta_{r}\right)$ - эффективный заряд ядра для электрона $r$-й группы, $V_{0}-$ объем элементарной ячейки, $c_{r}$ и $d_{r}-$ параметры, которые подбираем так, чтобы в дальнейших расчетах исчезли все члены, расходящиеся при $V \rightarrow \infty ; 5)$ учитывая, что функция $\widetilde{B}_{\kappa s}^{\left(r^{\prime}\right)}$ слабо отличается от функции $B_{\text {к }}^{(\text {*) }}$, используем для вычисления трех- и шестикратных интегралов кубатурную формулу Гаусса

$$
I \equiv \int d^{n} x f(x) p(x) \approx Q \sum_{i} f\left(x_{i}\right)
$$

где $p(x)$ - весовая функция, $f(x)$ - произвольная функция, $Q-$ коэффициент Кристоффеля, $\left\{x_{i}\right\}-$ узловые точки, $n=3$ или 6 . Легко показать, что

$$
Q \approx \tilde{I}\left[\sum_{i} \bar{f}\left(x_{i}\right)\right]^{-1}
$$

где $I$ - точное значение интеграла типа (6), в котором подынтегральная функция $\bar{f}(\boldsymbol{x}) p(\boldsymbol{x})$ слабо отличается от выражения $f(\boldsymbol{x}) p(\boldsymbol{x})$. 
Функция (1а) будет приближенным решением одноэлектронного уравнения с потенциальной энергией (5), если $\omega_{r}=\left(Z-\delta_{r}\right) / n_{r}$, где $n_{r}$ - главное квантовое число.

Используя формулу (22) из $\left[{ }^{4}\right]$ и формулы (1)-(7), получаем в принятых приближениях и во втором приближении теории возмущений следующее выражение для энергии электронов твердого тела:

$$
\begin{aligned}
& E^{(e)}=-\frac{\mathrm{e}_{0}^{2} N_{y}}{a_{0}} \sum_{r=1}^{\tilde{n}-1}\left[\omega_{r, 0}^{2}+Z_{r}\left(Z / n_{r}-\omega_{r, 0}\right) \omega_{r, 0}\right] R_{r}+\mathrm{e}_{0}^{2} a_{0} \sum_{\kappa s}^{\{K\}} k^{2} / 2+ \\
& +\sum_{\substack{i=1 \\
(i \leqslant j)}}^{\tilde{n}} \sum_{j=1}^{\tilde{n}}\left[X_{1, \mathrm{ky}, \text { reg }}^{\left(B_{i} B_{j}\right)}+X_{1, \text { oб M }}^{\left(B_{i} B_{j}\right)}\right] R_{i} R_{j}+X_{2}^{(B)}+X_{2}^{(K)}+X_{\infty}
\end{aligned}
$$

где $a_{0}-$ радиус первой орбиты Бора, $\omega_{r 0}=\omega_{r} a_{0}, Z_{r}-$ число электро.нов $r$-й группы, приходящихся на один узел решетки,

$$
R_{r}=C_{\mathrm{K} r}^{2} \sum_{i=1}\left|B_{\mathrm{K} s}^{(r)}\right|_{\mathrm{x}_{i}}^{2}\left[\sum_{i=1}\left|B_{\mathrm{K} s}^{(r)}\right|^{2}\right]_{\mathrm{x}_{t}}^{-1}
$$

- корректирующий коэффициент

$\left(R_{\tilde{n}}=1\right), X_{1, \mathrm{ky} л, \text { reg }}^{\left(B_{B_{0}} B_{j}\right)}$ и $X_{1, \text { об } \mathrm{M}}^{\left(B_{i} B_{t}\right)}$ соответственно регулярная часть кулоновской энергии и обменная энергия взаимодействия электронов $i$-й группы с электронами $j$-й группы в старом представлении,

$$
X_{2}^{(K)}=-32 \pi^{2} Z^{2} e_{0}^{2}\left(a_{0} V_{0}^{2}\right)^{-1} \sum_{K s}^{\{K\}} \sum_{g \neq 0}|g|^{-4}\left(g^{2}-2 k g\right)^{-1}
$$

- корреляционная энергия внешних электронов, $g$ - радиус-вектор узла в обратной решетке, $X_{2}^{(B)}$ - корреляционная энергия внутренних электронов, $X_{\infty}-$ сумма всех членов, расходящихся при $V \rightarrow \infty$.

Минимизируя выражение (8) для $E^{(e)}$ при условии, что внешние электроны занимают область $k$-пространства, ограниченную сверху поверхностью Ферми, определяем энергию основного состояния электронов в твердом теле, равновесные значения параметров $\omega_{r}$ и постоянные решетки. Из (8) легко определяется выражение для энергетического электронного спектра твердого тела:

$$
\begin{aligned}
& E_{\mathrm{K} s}^{(e)}=\frac{\mathrm{e}_{0}^{2} a_{0} k^{2}}{2}-\frac{\mathrm{e}_{0}^{2}}{2 \pi}\left[\frac{k_{F}^{2}-k^{2}}{2|k|} \ln \frac{k_{F}+|k|}{\left|k_{F}-\right| k||}-\frac{k_{0}^{2}-k^{2}}{2|k|} \ln \frac{k_{0}+|k|}{\left|k_{0}-\right| k||}+\right. \\
& \left.+k_{F}-k_{0}\right]-32 \pi^{2} Z^{2} \mathrm{e}_{0}^{2}\left(a_{0} V_{0}^{2}\right)^{-1} \sum_{\mathrm{g} \neq 0}|g|^{-4}\left(g^{2}-2 k g\right)^{-1}+X_{\infty}^{(2)} / N_{y}+X_{\mathrm{K}}
\end{aligned}
$$

где $k_{F}$ и $k_{0}$ - радиусы сферических поверхностей, аппроксимирующих внешнюю и внутреннюю поверхности, ограничивающие область $k$-пространства, занятую внешними электронами; $X_{\infty}^{(K)}-$ доля расходящихся при $V \rightarrow \infty$ членов, приходящихся на внешние электроны:

$$
\sum_{\mathrm{K} s}^{\{K\}} X_{\mathrm{K}}=\sum_{j=1}^{\tilde{n}-1}\left[X_{1, \mathrm{Ky} J, \text { reg }}^{\left(K B_{j}\right)}+X_{1, \mathrm{oOM}}^{\left(K B_{j}\right)}\right] R_{j} / 2 \quad\left(K=B_{\tilde{n}}\right):
$$




\section{2. Баланс членов, расходящихся при $V \rightarrow \infty$}

Вычислим выражения $X_{\infty}$ и $X_{\infty}^{(K)}$, входящие в формулы (8) и (11). Для этого предположим, что валентная зона в нормальном металле заполнена электронами только на $50 \%$. В этом случае в выражение для энергии твердого тела входят следующие расходящиеся при $V \rightarrow \infty$ члены:

1. Энергия кулоновского взазимодействия остовных электронов с внешними

$$
\underset{1, \text { кул }}{(\text { Кв })}=\sum_{r} \mathrm{e}_{0}^{2} N_{y} Z_{r} V_{0}^{-1} \int d^{3} x|x|^{-1}
$$

2. Энергия кулоновского взаимодействия узлов решетки

$$
X_{3}=\mathrm{e}_{0}^{2} Z^{2} N_{y} 2^{-1} \sum_{1 \neq 0}|l|^{-1} .
$$

3. Энергия кулоновского взаимодействия внешних электронов между собой

$$
\underset{1, \text { кул }}{(\text { кK })}=\mathrm{e}_{0}^{2} N_{y}\left(2 V_{0}\right)^{-1} \int d^{3} x|x|^{-1} .
$$

4. Энергия кулоновского взаимодействия внешних электронов с решеткой

$$
X_{5, \infty}^{(K)}=-\mathrm{e}_{0}^{2} Z N_{y} V_{0}^{-1} \int d^{3} x|x|^{-1}
$$

5. Нерегулярная часть кулоновского взаимодействия внутренних электронов с решеткой

$$
\begin{aligned}
& X_{5, \infty}^{(B)}=\mathrm{e}_{0}^{2} Z \sum_{r} \sum_{\mathrm{K} s}^{\left\{B_{r}\right\}}\left[-\sum_{1 \neq 0} C_{r}^{2}|l|^{-1}+A_{\mathrm{Kr} r}^{2} V_{0}^{-1} \sum_{\mathrm{g} \neq 0} B_{r}^{2}\left(k+g, \omega_{r}\right) \times\right. \\
&\left.\times\left(2 \sum_{1 \neq 0}|l|^{-1}-V_{0}^{-1} \int d^{3} x|x|^{-1}\right)\right]
\end{aligned}
$$

где

$$
B_{r}\left(k, \omega_{r}\right)=\int d^{3} z \Pi_{r}(z) \mathrm{e}^{-\omega_{r}|\mathrm{z}|} \mathrm{e}^{i \kappa z}, \quad C_{r}^{2}=\left\langle C_{\mathrm{K} r}^{2}\right\rangle .
$$

6. Нерегулярная часть энергии кулоновского взаимодействия внутренних электронов между собой $\left(\underset{1, \text { кул }}{\left(X_{1}\right)}\right)_{\infty}=\sum_{r} \underset{r^{\prime}}{\sum}\left(X_{1, \mathrm{k} y}^{\left(B_{r^{\prime}} B_{r^{\prime}}\right)}\right)_{\infty}$.

Итак, часть энергии нормального металла, состоящая из всех членов, расходящихся при $V \rightarrow \infty$, равна

$$
\begin{gathered}
X_{\infty}=\mathrm{e}_{0}^{2} N_{y}\left[Z^{2} / 2 \sum_{1 \neq 0}|l|^{-1}+\left(2^{-1}-Z+\sum_{r} Z_{r}\right) V_{0}^{-1} \int d^{3} x|x|^{-1}\right]+ \\
+X_{5, \infty}^{(B)}+\left(X_{1, \text { кул }}^{(\text {Вв) }}\right)_{\infty} .
\end{gathered}
$$

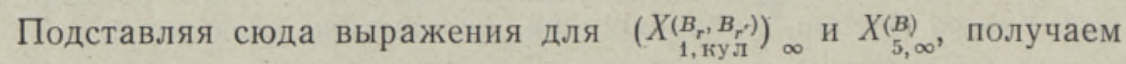

$$
X_{\infty}=\mathrm{e}_{0}^{2} N_{y}\left(-1 / 2+\varepsilon_{\infty}\right)\left(V_{0}^{-1} \int d^{3} x|x|^{-1}-\sum_{1 \neq 0}|l|^{-1}\right),
$$

где

$$
\varepsilon_{\infty}=V^{-1} \sum_{r} \sum_{\mathrm{K} s}^{\left\{B_{r}\right\}} A_{\mathrm{K} s}^{2} \sum_{\mathrm{g} \neq 0} B_{r}^{2}\left(k+g, \omega_{r}\right)\left(\sum_{r^{\prime}} Z_{r^{\prime}} C_{r^{\prime}}^{2}-Z\right)-
$$




$$
-V^{-2} \sum_{r r^{\prime}} \sum_{\mathrm{K} s}^{\left\{B_{r}\right\}} \sum_{\mathrm{K}^{\prime} s^{\prime}}^{\left\{B_{r^{\prime}}\right\}} A_{\mathrm{K} s}^{2} A_{\mathrm{K}^{\prime} g^{\prime}}^{2} \sum_{\mathrm{g}^{\prime} \neq 0} \sum_{\mathrm{g}^{\prime \prime} \neq 0} B_{r}^{2}\left(\boldsymbol{g}^{\prime}, \omega_{r}\right) B_{r}^{2}\left(\boldsymbol{k}^{\prime}+g^{\prime \prime}, \omega_{r^{\prime}}\right) .
$$

На долю внешних электронов приходится часть энергии $X_{\infty}$, равная

$$
X_{\infty}^{(K)}=\left(X_{1, \mathrm{Ky} \Omega}^{(K)}\right)_{\infty}+2^{-1} \sum_{r}\left(\underset{1, \text { Ку } \Omega}{\left(B_{r} K\right)}\right)_{\infty}+X_{5, \infty}^{(K)}+X_{3} / Z,
$$

что согласно (12)-(15) можно переписать так:

$$
X_{\infty}^{(K)}=-\mathrm{e}_{0}^{2} Z N_{y}\left(V_{0}^{-1} \int d^{3} x|x|^{-1}-\sum_{1 \neq 0}|l|^{-1}\right) / 2 .
$$

Согласно (18)-(21) на остовные электроны приходится энергия

$$
X_{\infty}^{(B)}=X_{\infty}-X_{\infty}^{(K)}=\mathrm{e}_{0}^{2} N_{y}\left[(Z-1) / 2+\varepsilon_{\infty}\right]\left(V_{0}^{-1} \int d^{3} x|x|^{-1}-\sum_{1 \neq 0}|l|^{-1}\right) .
$$

Отсюда следует, что на $r$-ю группу остовных электронов приходится следующая часть энергии $X_{\infty}$ :

$$
X_{\infty}^{\left(B_{r}\right)}=\mathrm{e}_{0}^{2} N_{y}\left(Z_{r} / 2+\varepsilon_{\infty} Z_{r} /(Z-1)\right)\left(V_{0}^{-1} \int d^{3} x|x|^{-1}-\sum_{1 \neq 0}|l|^{-1}\right) .
$$

\section{3. Электронная структура лития с ОЦК решеткой}

Электронная система ОЦК лития состоит из двух групп: а) $2 N_{y}$ остовных электронов $B$ (бывшие $1 s$-электроны); б) $N_{y}$ коллективизированных (внешних) электронов $K$ (бывшие $2 s$-электроны). Электроны группы $B$ занимают в $k$-пространстве объем, ограниченный сверху поверхностью, которая аппрокеимируется сферической поверхностью радиуса $k_{0}=4,91 / a$, где $a-$ постоянная решетки.

Для электронов группы $K$ аналогичный радиус равен $k_{F}=5,62 / a$. Одноэлектронная функция $B_{\text {кs }}^{(1)}$ выражается формулой $(2)$, где

$$
\varphi_{1 s}(x)=D \mathrm{e}^{-\omega|x|}, \quad D=\sqrt{\omega^{3} / \pi},
$$

$\omega$ - вариационный параметр.

Во втором приближении теории возмущений из (8) при $\tilde{n}=2$ получаем энергию электронов ОЦК лития:

$$
\begin{aligned}
& E^{(e)}=\sum_{\mathrm{K} s}^{\{B\}}\left(\widetilde{B}_{\mathrm{K} s},\left[\hat{T}-\mathrm{e}_{0}^{2} Z L\left(c_{r}, d_{r}\right)\right] \widetilde{B}_{\mathrm{Ks}}\right)+\sum_{\mathrm{\kappa} s}^{\{K\}}\left(K_{\mathrm{K} s},\left[\hat{T}-\mathrm{e}_{0}^{2} Z L(0,1)\right] K_{\mathrm{K} s}\right)+ \\
& +X_{1, \text { Кул }, \text { reg }}^{(B B)}+X_{1, \text { обм }}^{(B B)}+\underset{1, \text { Кул, reg }}{X_{1, \text { обм }}^{(K B)}}+\underset{1, \text { обм }}{X_{1}^{(K B)}}+\underset{2}{(K K)}+X_{2}^{(B)}+X_{\infty}^{(K)}+X_{\infty}
\end{aligned}
$$

где $\hat{T}$ - одноэлектронный оператор кинетической энергии, $\quad X(\boldsymbol{B}$, кул и $X_{1, \text { обм }}^{(B в)}-$ кулоновская и обменная энергии остовных (внутренних) электронов, $X_{1, \text { обм }}^{(\text {кв) }}$ - энергия обменного взаимодействия остовных электронов с внешними. Учитывая, что в данном случае функции $\left\{B_{\kappa s}\right\}$ описывают $1 s$-состояния, вычислим выражения, входящие в (25).

В ы ч слени е вы р а жения $\quad\left(\widetilde{B}_{\mathrm{Ks}},\left[\hat{T}-\mathrm{e}_{0}^{2} Z L\left(c_{r}, d_{r}\right)\right] \widetilde{B}_{\mathrm{Ks}}\right) \equiv I_{1}$. В данном случае имеем

$$
p(x)=\hat{T}-\mathrm{e}_{0}^{2} Z L\left(c_{r}, d_{r}\right), \quad f(x)=\left|\widetilde{B}_{\mathrm{K} s}(x)\right|^{2}, \quad \bar{f}(x)=\left|B_{\mathrm{Ks}}(x)\right|^{2} .
$$

Непосредственное интегрирование дает 


$$
\tilde{I}_{1}=\left(B_{\mathrm{Ks}},\left[\hat{T}-\mathrm{e}_{0}^{2} Z L\left(c_{r}, d_{r}\right)\right] B_{\mathrm{Ks}}\right)=-\frac{\mathrm{e}_{0}^{2} \omega_{0}}{a_{0}}\left(3-\frac{\omega_{0}}{2}\right) .
$$

Учитывая (26) и (27), из (6) и (7) получаем

$$
I_{1} \doteq-\frac{\pi \mathrm{e}_{0}^{2} a_{0}^{2}}{\omega_{0}^{2}}\left(3-\frac{\omega_{0}}{2}\right)\left[\widetilde{B}_{\mathrm{Ks}}\right]_{x=0}^{2}
$$

Вычи слени е кулоновской энергии $I_{2} \equiv \underset{1, \text { кул, reg }}{\left(B_{1}\right.}$.

Имеем

$$
\begin{gathered}
p\left(x_{1}, x_{2}\right)=\left|x_{1}-x_{2}\right|^{-1}, \quad f\left(x_{1}, x_{2}\right)=\mathrm{e}_{0}^{2} N_{y}\left|\widetilde{B}_{\mathrm{Ks}}\left(x_{1}\right) \widetilde{B}_{\mathrm{Ks}}\left(x_{2}\right)\right|^{2}, \\
\bar{f}\left(x_{1}, x_{2}\right)=\mathrm{e}_{0}^{2} N_{y}\left|B_{\mathrm{Ks}}\left(x_{1}\right) B_{\mathrm{Ks}}\left(x_{2}\right)\right|^{2} .
\end{gathered}
$$

Элементарный расчет дает

$$
\tilde{I}_{2}=\mathrm{e}_{0}^{2} N_{y}\left\{\int d^{3} x_{1} \int d^{3} x_{2} \frac{\left|B_{\mathrm{Ks}}\left(x_{1}\right) B_{\mathrm{Ks}}\left(x_{2}\right)\right|^{2}}{\left|x_{1}-x_{2}\right|}\right\}_{r e g}=\frac{1,25 \mathrm{e}_{0}^{2} \omega_{0}}{a_{0}}
$$

Учитывая (29)-(31), из (6) и (7) получаем

$$
I_{2}=[\underset{1, \text { кул }}{X(B)}]_{\text {reg }}=1,25 \pi^{2} a_{0}^{5} \mathrm{e}_{0}^{2} N_{y \omega_{0}^{-5}}\left|\widetilde{B}_{\mathrm{\kappa s}}\right|_{\mathrm{x}=0}^{4} .
$$

Аналогично находим, что

$$
X_{1, \text { обм }}^{(B)} \approx-0,625 \pi^{2} a_{0}^{5} \mathrm{e}_{0}^{2} N_{y} \omega_{0}^{-5}\left|\widetilde{B}_{\mathrm{Ks}}\right|_{\mathrm{X}=0}^{4}:
$$

В ы и слени е вы ражения $I_{3} \equiv \underset{1, \text { обм }}{(\text { BK) }}$ В данном случае имеем

$$
\begin{gathered}
p\left(x_{1}, x_{2}\right)=\left|x_{1}-x_{2}\right|^{-1}, \quad f\left(x_{1}, x_{2}\right)=-\frac{\mathrm{e}_{0}^{2}}{2 N_{y}} \sum_{{\mathrm{K} s \mathrm{~K}^{\prime}}^{\prime}}\left(\widetilde{B}_{\mathrm{K} s}^{*} K_{\mathrm{K}^{\prime} s}\right)_{1}\left(K_{\mathrm{K}^{\prime} s}^{*} \widetilde{B}_{\mathrm{K} s}\right)_{2}, \\
\bar{f}\left(x_{1}, x_{2}\right)=-\frac{\mathrm{e}_{0}^{2}}{2 N_{y}} \sum_{\mathrm{K} s \mathrm{~K}^{\prime}}\left(B_{\mathrm{Ks}}^{*} K_{\mathrm{K}^{\prime} s}\right)_{1}\left(K_{\mathrm{K}^{\prime} s}^{*} B_{\mathrm{K} s}\right)_{2} .
\end{gathered}
$$

Из элементарного расчета следует

$$
\begin{gathered}
I_{3}=-\mathrm{e}_{0}^{2}\left(2 N_{y}\right)^{-1} \sum_{{\mathrm{K} s \mathrm{~K}^{\prime}}^{\prime}} \int d^{3} x_{1} \int d^{3} x_{2}\left(B_{\mathrm{K} s}^{*} K_{\mathrm{K}^{\prime} s}\right)_{1}\left(K_{\mathrm{K}^{\prime} s}^{*} B_{\mathrm{K} s}\right)_{2}\left|x_{1}-x_{2}\right|^{-1}= \\
=-8 \pi \mathrm{e}_{0}^{2} N_{y}\left(3 a^{3} \omega^{2} Y\right)^{-1}\left(8 / Y^{2}+4 / Y+3\right)
\end{gathered}
$$

где $Y=1+\mathbf{k}^{2} / \omega^{2}$. Используя формулы $(34)-(36)$, из (6) и (7) находим

$$
I_{3} \equiv X_{1,00 \mathrm{M}}^{(B K)}=-8 \pi^{2} \mathrm{e}_{0}^{2} N_{y}\left(3 \omega^{5} a^{3} Y\right)^{-1}\left(8 / Y^{2}+4 / Y+3\right)\left|\widetilde{B}_{\mathrm{K} s}\right|_{\mathrm{x}=0}^{2} .
$$

Полученные выше результаты можно уточнить, используя для этого более сложные кубатурные формулы Гаусса. Выражения (28), (32), (33) и (37) показывают, что в первом приближении формулы метода, рассмотренного в разделе 1 , отличаются от аналогичных формул простой модели, в которой внешние электроны описываются плоскими волнами, наличием в них поправочных коэффициентов типа $\left|\tilde{B}_{\mathrm{\kappa} s}^{(r)}\right|_{\mathrm{x}=0}^{2}$. Легко показать, что в общем случае каждая группа остовных электронов имеет свой поправочный коэффициент $\left|\widetilde{B}_{\text {кs }}^{(r)}\right|^{2}$. Заметим, что предложенный в разделе 1 метод вычисления интегралов обеспечивает 
сведение объема вычислений к минимуму, так как в этом методе вычисляемые интегралы не расщепляются на огромное количество более «мелких» интегралов.

Подставляем выражения (28), (32), (33) и (37) в формулу (25) и переходим к приближению, когда в предпоследнем члене в (25) суммирование производится по первым двум координационным сферам в обратной решетке. Минимизируя полученное выражение для $E^{(e)}$, находим энергию основного состояния лития ОЦК структуры (на один узел решетки) $E=-7,52 \mathrm{e}_{0}^{2} / a_{0}$ и постоянную решетки $a=3,86 \AA$. Эксперимент для этих величин дает: $E=-7,51 \mathrm{e}_{0}^{2} / a_{0}, a=3,509 \AA$.

\section{4. Электронная структура натрия с ОЦК решеткой}

Электронная система ОЦК натрия состоит из четырех групп: а) $2 N_{y}$ остовных электронов $B_{1}$ (бывшие $1 s$-электроны); б) $2 N_{y}$ остовных электронов $B_{2}$ (бывшие $2 s$-электроны); в) $6 N_{y}$ остовных электронов $B_{3}$ (бывшие $2 p$-электроны); г) $N_{y}$ коллективизированных (внешних) электронов $B_{4}=K$ (бывшие $3 s$-электроны).

Әлектроны группы $B_{1}$ занимают в $k$-пространстве объем, 'сграниченный сверху поверхностью, которая аппроксимируется сферической поверхностью радиуса $k_{1}=4,91 / a$. Для электронов групп $B_{2}, B_{3}$ и $B_{4}$ аналогичные радиусы равны $k_{2}=6,187 / a, k_{3}=8,398 / a$ и $k_{F}=8,669 / a$ соответственно. Функции $B_{\text {кs }}^{(r)}$ выражаются формулой (2), при этом

$$
\begin{gathered}
\varphi_{1 s}(x)=D_{1 s} \mathrm{e}^{-\omega_{t s}|x|}, \quad \varphi_{2 s}(x)=D_{2 s}\left(2-\tilde{\omega}_{2 s}|x|\right) \mathrm{e}^{-\omega_{2 s}|x|}, \\
\varphi_{2 p_{t}}(x)=D_{2 p} \cdot(x)_{i} \mathrm{e}^{-\omega_{2 p}|x|} \quad(i=1,2,3),
\end{gathered}
$$

где

$$
\begin{gathered}
D_{1 s}^{2}=\omega_{1 s}^{3} / \pi, \quad D_{2 s}^{2}=\omega_{2 s}^{3}\left[1-1,5 \tilde{\omega_{2 s}} / \omega_{2 s}+3\left(\tilde{\omega_{2 s}} / \omega_{2 s}\right)^{2} / 4\right]^{-1} /(4 \pi), \\
D_{2 p}^{2}=\omega_{2 p}^{5} / \pi
\end{gathered}
$$

$\omega_{1 s}, \omega_{2 s}, \tilde{\omega}_{2 s}$ и $\omega_{2 p}-$ вариационные параметры $\left(\tilde{\omega}_{2 s} / \omega_{2 s}\right)=2$. Функции $\varphi_{r}(x)$ взаимно ортогональны. Переходя ко второму приближению и используя формулу (1a), получаем одноэлектронные волновые функции остовных электронов

$$
\widetilde{B}_{\mathrm{K} s}^{(r)} \approx B_{\mathrm{K} s}^{(r)}-\left(K_{\mathrm{K}^{\prime} s^{\prime}} \mid B_{\mathrm{K} s}^{(r)}\right) K_{\mathrm{K}^{\prime} s^{\prime}}
$$

где $r=1 s, 2 s, 2 p_{x}, 2 p_{y}$ или $2 p_{z}$, а волновой вектор $\boldsymbol{k}^{\prime}$ принадлежит зоне Бриллюэна, занятой внешними электронами, $\boldsymbol{k}^{\prime}=\boldsymbol{k}+\boldsymbol{g}$. При этом из условия нормировки функций $\widetilde{B}_{\mathrm{ks}}^{(r)}$ получаем

$$
\begin{gathered}
C_{\mathrm{K}, 1 s}^{2}=\left[1-\frac{128 \pi}{a^{3} \omega_{1 s}^{3} Y_{1 s}^{4}}\right]^{-1}, \quad C_{\mathrm{K}, 2 s}^{2}=\left[1-\frac{128 \pi\left(2-4 / Y_{2 s}\right)^{2}}{a^{3} \omega_{2 s}^{3} Y_{2 s}^{4}}\right]^{-1}, \\
C_{\mathrm{K}, 2 p}^{2}=\left[1-\frac{2048 \pi\left\langle k^{2}\right\rangle}{3 a^{3} \omega_{2 p}^{5} Y_{2 p}^{6}}\right]^{-1}, \quad A_{\mathrm{Kr} r}=C_{\mathrm{kr}} D_{r},
\end{gathered}
$$

где

$$
Y_{r}=1+\left\langle k^{2}\right\rangle / \omega_{r}^{2}, \quad\left\langle k^{2}\right\rangle=72,8312 / a^{2} .
$$

Энергия электронов ОЦК натрия.в нулевом приближении теории возмущений равна 


$$
\begin{aligned}
E_{0}^{(e)}= & -\left(\mathrm{e}_{0}^{2} N_{y} / a_{0}\right)\left(\omega_{1 s, 0}^{2} R_{1 s}+\omega_{2 s, 0}^{2} R_{2 s}+3 \omega_{2 p, 0}^{2} R_{2 p}\right)+ \\
& +\left(\mathrm{e}_{0}^{2} / a_{0}\right) \sum_{\kappa s}^{\{K\}}\left[a_{0}^{2} k^{2} / 2-\gamma\left(k_{x}, k_{y}, k_{z}\right)\right]
\end{aligned}
$$

где

$$
\begin{gathered}
R_{1 s}=C_{\mathrm{K}, 1 s}^{2}\left|\widetilde{B}_{\mathrm{K} s}^{(1 s)}\right|_{\mathrm{x}=0}^{2} /\left|B_{\mathrm{Ks}}^{(1 s)}\right|_{\mathrm{x}=0}^{2}=C_{\mathrm{K}, 1 s}^{2}\left[1-16 \pi /\left(a^{3} \omega_{1 s}^{3} Y_{1 s}^{2}\right)\right], \\
R_{2 s}=C_{\mathrm{K}, 2 s}^{2}\left|\widetilde{B}_{\mathrm{K} s}^{(2 s)}\right|_{\mathrm{x}=0}^{2} /\left|B_{\mathrm{Ks}}^{(2 s)}\right|_{\mathrm{x}=0}^{2}=C_{\mathrm{K}, 2 s}^{2}\left[1-16 \pi\left(2-4 / Y_{2 s}\right) /\left(a^{3} \omega_{2 s}^{3} Y_{2 s}^{2}\right)\right]^{2}, \\
R_{2 p}=C_{\mathrm{K}, 2 p}^{2} \sum_{i=1}^{6}\left|B_{\mathrm{K} s}^{(2 p)}\right|_{\mathrm{x}_{t}}^{2} / \sum_{i=1}^{6}\left|B_{\mathrm{K} s}^{(2 p)}\right|_{\mathrm{x}_{t}}^{2}=C_{\mathrm{K}, 2 p}^{2}\left[1-\frac{128 \mathrm{e}\left\langle k^{2}\right\rangle}{3 a^{3}\left(\omega_{2 p}^{5} Y_{2 p}^{3}\right.}+\right.
\end{gathered}
$$$$
\left.+\frac{\left\langle k^{2}\right\rangle}{3}\left(\frac{64 \pi \mathrm{e}}{a^{3} \omega_{2 p}^{2} Y^{3}}\right)^{2}\right],
$$

$x_{1}=\left(\omega_{2 p}^{-1}, 0,0\right), \quad x_{2}=\left(-\omega_{2 p}^{-1}, 0,0\right), \quad x_{3}=\left(0, \omega_{2 p}^{-1}, 0\right), \quad x_{4}=\left(0,-\omega_{2 p}^{-1}, 0\right)$, $x_{5}=\left(0,0, \omega_{2 p}^{-1}\right), x_{6}=\left(0,0,-\omega_{2 p}^{-1}\right),|x|=\omega_{2 p}^{-1}-$ точка, в которой функция $|x| \exp \left(-\omega_{2 p}|x|\right)$ достигает максимума. При выводе формулы (46) учтено, что $\mathrm{e}^{-2 \omega_{2 p}|1|} \ll \mathrm{e}^{-1}, \sin \left(k_{x}^{\prime} / \omega_{2 p}\right) \approx k_{x}^{\prime} / \omega_{2 p}$.

Добавки к энергии электронов в первом и втором приближениях теории возмущений соответственно равны

$$
\begin{gathered}
E_{1}^{(e)}=-\left(\mathrm{e}_{0}^{2} N_{y} / a_{0}\right) \cdot\left[2\left(11-\omega_{1 s, 0}\right) \omega_{1 s, 0} R_{1 s}+\left(11-2 \omega_{2 s, 0}\right) \omega_{2 s, 0} R_{2 s}+\right. \\
\left.+3\left(11-2 \omega_{2 p, 0}\right) \omega_{2 p, 0} R_{2 p}\right]+\left(\mathrm{e}_{0}^{2} / a_{0}\right) \sum_{\mathrm{\kappa} s}^{\{K\}} \gamma\left(k_{x}, k_{y}, k_{z}\right), \\
E_{2}^{(e)}=X_{1, r e g}+X_{2}+X_{\infty} .
\end{gathered}
$$

Энергию регулярной части кулоновского и обменного взаимодействий можно представить так:

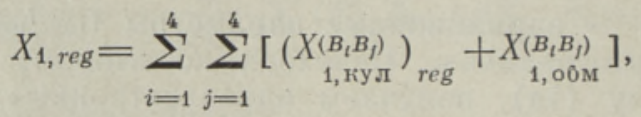

где

$$
\begin{gathered}
X_{1, \text { обм }}^{(K K)}=-\frac{\mathrm{e}_{0}^{2}}{2 \pi} \sum_{\text {Ks }}^{\{K\}}\left[\frac{k_{F}^{2}-k^{2}}{2|k|} \ln \frac{k_{F}+|k|}{\left|k_{F}-\right| k||}-\frac{k_{3}-k^{2}}{2|k|} \ln \frac{k_{3}+|k|}{\left|k_{3}-\right| k||}+\right. \\
\left.+k_{F}-k_{3}\right]=-0,2517 \frac{\mathrm{e}_{0}^{2} N_{y}}{a}
\end{gathered}
$$

- обменная энергия внешних электронов,

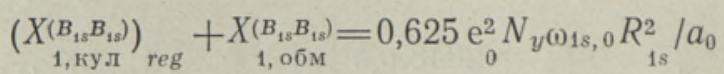

- регулярная часть кулоновской энергии и обменная энергия электронов группы $B_{1}$,

$$
\left.\underset{1, \text { кул }}{\left(X_{\text {reg }}^{\left(B_{23} B_{2 s}\right)}\right.}\right)_{\substack{1, \text { обм } \\\left(B_{28} B_{2 s}\right)}}=0,30079 \mathrm{e}_{0}^{2} N_{y}\left(\omega_{2 s, 0} R_{2 s}^{2} / a_{0}\right.
$$

- регулярная часть кулоновской энергии и обменная энергия электронов группы $B_{2}$, 


$$
\left.\underset{1, \mathrm{Ky} J}{\left(X\left(B_{2 p} B_{2 p}\right)\right.}\right)_{r e g}+\underset{1, \text { об }}{\left(B_{2 p} B_{2 p}\right)}=5,2383 \mathrm{e}_{0}^{2} N_{y}\left(\omega_{2 p, 0} R_{2 p}^{2} / a_{0}\right.
$$

- регулярная часть кулоновской энергии и обменная энергия электронов группы $B_{3}$,

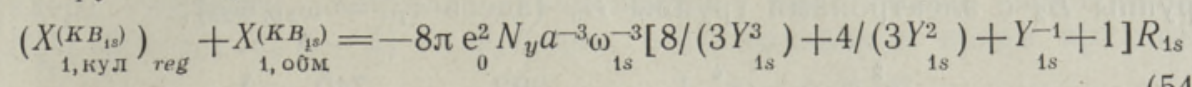

- энергия взаимодействия внешних электронов с электронами группы $B_{1}$,

$$
\begin{gathered}
\left(X_{1, \mathrm{Ky} J}^{\left(K B_{2}\right)}\right)_{\text {reg }}+X_{1, \text { o6 M }}^{\left(K B_{2 s}\right)}= \\
=\frac{4 \pi \mathrm{e}_{0}^{2} N_{y}}{a^{3} \omega_{2 s}^{2}}\left[3-\frac{2}{Y_{2 s}}\left(1+\frac{4}{3 Y_{2 s}}+\frac{4,2(6)}{Y_{2 s}^{2}}-\frac{96}{5 Y_{2 s}^{3}}+\frac{128}{5 Y_{2 s}^{4}}\right)\right] R_{2 s}
\end{gathered}
$$

- энергия взаимодействия внешних электронов с электронами группы $B_{2}$,

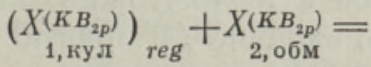

$$
\begin{aligned}
& =-\frac{4 \pi \dot{\mathrm{e}}_{0}^{2} N_{y}}{a^{3} \omega_{2 p}^{2}}\left[15+2\left(\frac{3}{Y_{2 p}}+\frac{4}{Y_{2 p}^{2}}+\frac{9,6}{Y_{2 p}^{3}}-\frac{96}{5 Y_{2 p}^{4}}+\frac{128}{5 Y_{2 p}^{5}}\right)\right] R_{2 p}
\end{aligned}
$$

- энергия взаимодействия внешних электронов с электронами группы $B_{3}$,

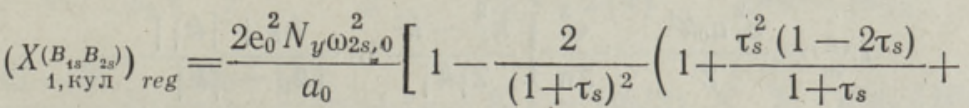

$$
\begin{aligned}
& \left.\left.+\frac{3 \tau_{s}^{3}\left(\tau_{s}-2\right)}{2\left(1+\tau_{s}\right)^{2}}+\frac{3 \tau_{s}^{4}}{\left(1+\tau_{s}\right)^{3}}\right)\right] R_{1 s} R_{2 s}
\end{aligned}
$$

- регулярная часть кулоновской энергии взаимодействия электронов группы $B_{1}$ с электронами группы $B_{2}$ (здесь $\left.\tau_{s}=\omega_{2 s} / \omega_{1 s}\right)$,

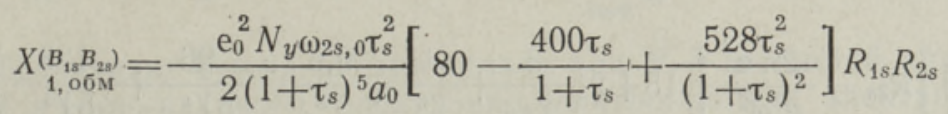

- обменная энергия взаимодействия электронов группы $B_{1}$ с электронами группы $B_{2}$,

$$
\left.\underset{1, \mathrm{Ky} J}{\left(X_{18}^{\left(B_{18} B_{2 p}\right.}\right)}\right)_{r e g}=6 \mathrm{e}_{0}^{2} N_{y} \omega_{2 p, 0}\left[1-\tau_{p}^{4}\left(3+\tau_{p}\right)\left(1+\tau_{p}\right)^{-5}\right] R_{1 s} R_{2 p} / a_{0}
$$

- регулярная часть кулоновской энергии взаимодействия электронов группы $B_{1}$ с электронами группы $B_{3}$ (здесь $\left.\tau_{p}=\omega_{2 p} / \omega_{1 s}\right)$,

$$
\underset{\substack{\left.X_{18} B_{2 p}\right) \\ 1,06 \mathrm{M}}}{X\left(B_{0}\right.}=-56 \mathrm{e}^{2} N_{y} \omega_{2 p, 0} \tau_{p}^{2}\left(1+\tau_{p}\right)^{-7} R_{1 s} R_{2 p} / a_{0}
$$

- обменная энергия взаимодействия электронов группы $B_{1}$ с электронами группы $B_{3}$,

$$
\left(X_{1, \mathrm{Ky} J}^{\left(B_{2 s} B_{2}\right)}\right)_{r e g}=\frac{6 \mathrm{e}_{0}^{2} N_{y} \omega_{2 p, 0}}{a_{0}}\left[1-\frac{\tau_{p s}^{4}}{\left(1+\tau_{p s}\right)^{4}}\left(1+\frac{3}{1+\tau_{p s}}+\right.\right.
$$




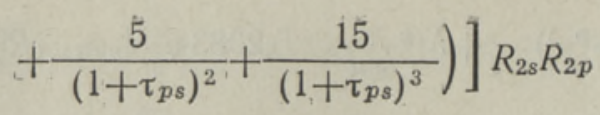

- регулярная часть кулоновской энергии взаимодействия электронов группы $B_{2}$ с электронами группы $B_{3}$ (здесь $\left.\tau_{p s}=\omega_{2 p} / \omega_{2 s}\right)$,

$$
\left.X_{\substack{\left(B_{2,} B_{2 p}\right) \\ 1,0 \sigma \mathrm{M}}}^{\left(1+\tau_{p s}\right)^{7} a_{0}}=-\frac{\mathrm{e}_{0}^{2} N_{y} \omega_{2 p, 0} \tau_{p s}^{4}}{\left(1+\tau_{p s}\right.}+\frac{740}{\left(1+\tau_{p s}\right)^{2}}\right] R_{2 s} R_{2 p}
$$

- обменная энергия взаимодействия электронов группы $B_{2}$ с электронами группы $B_{3}$.

Входящая в правую часть (48) корреляционная энергия внешних электронов натрия ОЦК структуры'равна

$$
X_{2}^{(K)} \approx-\frac{\mathrm{e}_{0}^{2}}{a_{0}} \sum_{\kappa s}^{\{K\}} \sum_{g \neq 0} \frac{32 \pi^{2} Z^{2}}{V_{0}^{2}|g|^{4}\left(g^{2}-2 k g\right)} \approx-0,52582 \frac{\mathrm{e}_{0}^{2} N_{y}}{a_{0}}
$$

Вклад остовных электронов в $X_{2}$ ничтожно мал. Поэтому мы им пренебрегаем. Выражение $X_{\infty}$, входящее в правую часть (48), вычисляется по формуле (18). Минимизируя выражение

$$
E^{(e)}=E_{0}^{(e)}+E_{1}^{(e)}+E_{2}^{(e)}
$$

где $E_{0}^{(e)}, E_{1}^{(e)}$ и $E_{2}^{(e)}$ представлены формулами $(43)-(48)$, находим энергию основного состояния электронов натрия ОЦК структуры.

Согласно (43), (47)-(50), (54)-(56) и (63) выражение

$$
\begin{gathered}
E_{\mathrm{Ks}}^{(K)}=\frac{\mathrm{e}_{0}^{2} a_{0} k^{2}}{2}-\frac{\mathrm{e}_{0}^{2}}{2 \pi}\left[\frac{k_{F}^{2}-k^{2}}{2|k|} \ln \frac{k_{F}+|k| \mid}{\left|k_{F}-\right| k \mid}-\right. \\
\left.-\frac{k_{3}^{2}-k^{2}}{2|k|} \ln \frac{k_{3}+|k|}{\left|k_{3}-\right| k||}+k_{F}-k_{3}\right]-\frac{4 \pi \mathrm{e}_{0}^{2}}{a^{3} \omega_{1 s}^{3}}\left(\frac{8}{3 Y_{1 s}^{3}}+\frac{4}{3 Y_{1 s}^{2}}+\right. \\
\left.+\frac{1}{Y_{1 s}}+1\right) R_{1 s}+\frac{2 \pi \mathrm{e}_{0}^{2}}{a^{3} \omega_{2 s}^{2}}\left[3-\frac{2}{Y_{2 s}}\left(1+\frac{4}{3 Y_{2 s}}+\frac{4,2(6)}{Y_{2 s}^{2}}-\frac{96}{5 Y_{2 s}^{3}}+\right.\right. \\
\left.\left.+\frac{128}{5 Y_{2 s}^{4}}\right)\right] R_{2 s}-\frac{2 \pi \mathrm{e}_{0}^{2}}{a^{3} \omega_{2 p}^{2}}\left[15+2\left(\frac{3}{Y_{2 p}}+\frac{4}{Y_{2 p}^{2}}+\frac{9,6}{Y_{2 p}^{3}}-\frac{96}{5 Y_{2 p}^{4}}+\frac{128}{5 Y_{2 p}^{5}}\right)\right] R_{2 p}- \\
-\left(\mathrm{e}_{0}^{2} / a_{0}\right) \sum_{\mathrm{g} \neq 0} \sum 3872 \pi^{2} /\left(V_{0}^{2}|g|^{4}\left(g^{2}-2 k g\right)\right)-X_{\infty}^{(K)} / N_{y},
\end{gathered}
$$

где $Y_{r}=1+\mathbf{k}^{2} / \omega_{r}^{2}$ дает энергетический электронный спектр натрия ОЦК структуры.

\section{5. Электронная структура жидкого металла}

Рассмотрим модель жидкого металла, в которой все остовные электронные состояния локализованы, а внешние электронные состояния частично локализованы и частично коллективизированы. При этом мы вводим новое представление, в котором внешние коллективизированные одноэлектронные состояния описываются плоскими волнами $K_{\text {кs }}$. Пусть в $k$-пространстве эти состояния занимают область, ограниченную двумя сферическими поверхностями с радиусами соответственно $k_{0}$ 
и $k_{F}$. Пंоверхность с радиусом $k_{F}-$ поверхность Ферми. Величину $k_{0}$ можно найти, минимизируя выражение для свободной энергии жидкого металла.

Введем обозначения: $\left\{\mathrm{R}_{l}\right\}-$ множество радиус-векторов узлов (ядер) жидкого металла, являющихся случайными величинами, $\left\{\widetilde{B}_{\mathrm{R}_{l}}^{(r)}\right\}(r=1, \ldots, \tilde{n}-2)-$ функции, описывающие в новом представлении внутренние локализованные электроны, $\left\{\widetilde{B}_{\mathrm{R}_{l}}^{(\tilde{n}-1)}\right\}-$ функции, описывающие внешние локализованные электроны в новом представлении. Итак, в новом-представлении ортонормированная система одноэлектронных функций $G$ состоит из плоских волн $K_{\text {гs }}$ и из локализованных функций $\left\{\widetilde{B}_{\mathrm{R}_{1}}^{(j)}\right\}$, где $j=1, \ldots, \tilde{n}-1$. Оператор энергии электронной системы жидкого металла выражается формулой, которую можно получить, если заменить в эффективных потенциальных энергиях, входящих в формулы (4) и (5), радиус-векторы $\{\boldsymbol{1}\}$ идеальной кристаллической решетки на радиус-векторы $\left\{\mathrm{R}_{l}\right\}$.

Используя этот оператор энергии и выполняя расчет, сходный с расчетом, выполненным в разделе 1, для энергии электронной системы жидкого металла получаем формулу

$$
E=\sum_{j=1}^{=k} E^{\left(B_{f}\right)}+\sum_{K s}^{\{K\}} E_{K s}^{(K)},
$$

где

$$
\begin{gathered}
E_{\mathrm{K} s}^{(K)}=\frac{e_{0}^{2}}{a_{0}}\left\{\frac{a_{0}^{2} k^{2}}{2}-\frac{a_{0} Z \tilde{S}}{2}-\frac{a_{0}}{2 \pi}\left[\frac{k_{F}^{2}-k^{2}}{2|k|} \ln \frac{k_{F}+|k|}{\left|k_{F}-\right| k||}-\right.\right. \\
\left.-\frac{k_{0}^{2}-k^{2}}{2|k|} \ln \frac{k_{0}+|k|}{\left|k_{0}-\right| k||}+k_{F}-k_{0}\right]-\left(a_{0} / 2\right) \sum_{j=1}^{\tilde{n}-1} \sum_{l}^{\left\{B_{t}\right\}} \int d^{3} x_{1} \int d^{3} x_{2} \times \\
\left.\times\left(\widetilde{B}_{\mathrm{R}_{l}}^{(j) *} K_{\mathrm{K} s}\right)_{1}\left(K_{\mathrm{Ks} s}^{*} \widetilde{B}_{\mathrm{R}_{l}}^{(j)}\right)_{2} \cdot\left|x_{1}-x_{2}\right|^{-1}\right\}
\end{gathered}
$$

- энергия коллективизированного электрона,

$$
\widetilde{S}=V_{0}^{-1} \int d^{3} x \cdot|x|^{-1}-\sum_{1 \neq 0}\left|R_{l}\right|^{-1}
$$

В (66) входит сумма расходящихся при $V \rightarrow \infty$ частей кулоновских энергий ядерно-ядерного, электронно-электронного и электронно-ядерного взаимодействий

$$
\begin{gathered}
X_{\infty}=\left(D \mathrm{e}_{0}^{2} / 2\right) \sum_{m \neq n}^{N_{y}} \sum^{N_{y}}\left|R_{m}-R_{n}\right|^{-1}-\left(D_{1} \mathrm{e}_{0}^{2} / 2 V_{0}\right) \sum_{m=1}^{N_{y}} \int d^{3} x\left|x-\boldsymbol{R}_{m}\right|^{-1}+ \\
+\left(D_{1}-D\right)\left(\mathrm{e}_{0}^{2} / 2 V_{0}^{2}\right) \int d^{3} x \int d^{3} x^{\prime} \cdot\left|x-x^{\prime}\right|^{-1},
\end{gathered}
$$

где $D$ и $D_{1}$ - коэффициенты, определяемые методом, рассмотренным в разделе 2 .

\section{6. Выводы}

Предложенный в данной работе метод расчета электронной структуры твердого тела объединяют с методом псевдопотенциала (МПП) две общие черты (см. $\left.\left[{ }^{6-9}\right]\right)$ : построение ортогонализированной системы функций из атомных функций и плоских волн; и трансформация урав- 
нения Шредингера к новому представлению, в котором потенциальная функция отличается от исходного выражения для потенциальной энергии (в МПП, как известно, эта потенциальная функция называется псевдопотенциалом) .

Однако наш метод существенно отличается от МПП. Во-первых, в методе ортогонализированных плоских волн и в МПП одноэлектронные функции остовных электронов остаются неизменными, а одноэлектронные функции внешних электронов, взятые в виде линейных комбинаций нескольких плоских волн, ортогонализируются к волновым функциям остовных электронов. В нашем методе имеет место обратная процедура: внешний электрон описывается одной плоской волной, а волновые функции остовных электронов ортогонализируются к плоской волне. Во-вторых, в МПП псевдопотенциал является функцией координат электрона. В нашем методе аналогичная функция для внешних электронов является функцией производных по координатам электрона. В-третьих, наш метод построен на более общих теоретических принципах и поэтому он более корректен, чем МПП. Последний, на наш взгляд, носит отпечаток частного, удачно придуманного искусственного приема. Остюда вытекают следующие преимущества нашего метода перед МПП. Первое. В МПП строят различного рода модельные псевдопотенциалы, зависящие от подгоночных параметров, которые определяют из эксперимента. В нашем методе параметры, входящие в соответствующую потенциальную функцию, определяют с помощью вариационного метода. В торое. В случае МПП необходимо решать систему уравнений для определения коэффициентов, входящих в линейную комбинацию плоских волн, являющуюся волновой функцией внешнего электрона. В нашем случае этот расчет не требуется, так как внешний электрон описывается одной единственной плоской волной. Третье. Поскольку перекрытие атомных функций внутренних электронов, взятых для разных узлов решетки, пренебрежимо мало, а в новом представлении внешние электроны описываются плоскими волнами, то все шестикратные кулоновские и обменные интегралы вычисляются элементарно, что сводит объем вычислительных работ к минимуму. Поэтому отпадает необходимость в переходе к весьма приближенному методу Слэтера вычисления обменных интегралов. Четвертое. Энергия электронов в твердом теле во втором порядке теории возмущений в нашем методе содержит, кроме кулоновской и обменной энергии, еще и корреляционную энергию $X_{2}$. Поэтому наш метод фактически выходит за рамки приближения Хартри-Фока. В МПП обменная и корреляционная энергии входят в зависимый от подгоночных параметров псевдопотенциал. Формула (5.4) в $\left[{ }^{2}\right]$ (или формула (3.15) в $\left[{ }^{3}\right]$ ) описывает корреляционную энергию электронов в нашем методе. Используя эту формулу, мы получили предпоследний член в правой части (8), т. е. корреляционную энергию внешних электронов. Расчет показал, что корреляционная энергия остовных электронов гораздо меньше соответствующей величины для внешних электронов. П я тое. Энергетический электронный спектр твердого тела определяется в нашем методе в аналитической замкнутой форме, удобной для вычисления физических свойств твердых тел. Шестое. Так как атомные волновые функции остовных электронов, взятые для разных узлов решетки, перекрываются слабо, а внешние электроны в новом представлении описываются плоскими волнами, то рассматриваемый здесь метод легко обобщается на неупорядоченные структуры (в частности, на аморфные тела и жидкие.металлы).

Заметим, что в рассматриваемом здесь методе, переходя к более высоким приближениям теории возмущений, чем второе, мы можем 
получить более точное выражение для энергетического электронного спектра твердого тела, чем приведенное в данной работе.

Предложенный здесь метод можно обобщить на другие классы твердых тел. В частности, при распространении его на полупроводники, необходимо новое представление выбирать так, чтобы электроны в зоне проводимости описывались плоскими волнами. В переходных металлах (в отличие от нормальных) в новом представлении только часть внешних электронов (бывшие $s$ - или $p$-электроны) описывается плоскими волнами; остальная часть этих электронов (бывшие $d$ - или $f$-электроны) описывается функциями типа (1).

Кроме того, наш метод позволяет построить квантовую теорию хрупкого разрушения материалов и квантовую теорию прочностных свойств материалов.

\section{ЛИТЕРАТУРА}

1. Куиниренко А. Н., Филин В. Г. Докл. АН УССР, Сер. А. № 1, 58-61 (1979).

2. Куиниренко А. Изв. АН ЭССР. Физ. Матем., 31. № 1, $38-48$ (1982).

3. Кушниренко А. Н. Препринт ИПМ АН УССР. № 1. Киев. 1979.

4. Кчиниренко А. Н. Изв. ВУЗов. Физикя, № 6. 11-15 (1980)

5. Кушниренко А. Н. Препринт ИПМ АН УССР, № 1. Кнев, 1976.

6. Herring, C. Phvs. Rev., 57, 1169 (1940).

7. Phillips, J. C., Kleinman, L. Phys. Rev., 116, 287 (1959).

8. Харрисон У. Псевдопотенцналы в теории металлов. М., «Мир». 1968.

9. Хейне В., Коэн М., Уэйр Д. Теория псевдопотенциала. М., «Мир», 1973.

Ннститут проблем материаловедения Академии наук украинской ССР
Поступнла в редакцию 25 ноября 1981

После переработки 3 сентября 1982

\section{A. KUSNIRENKO}

\section{NORMAALMETALLIDE KVANTELEKTRODUNAAMILINE MUDEL}

Artiklis on esitatud uudne normaalmetalli mudel, milles tahket keha käsitatakse kvantelektron- ia -tuumavälia süsteemina, kusjuures nimetatud väliade vastastikune mōju teostub elektromagnetvälia kaudu. See võimaldab tänselt arvutada normaalmetalli elektronide korrelatsioonienergiat. Arvutustööde mahu vähendamise eesmärgil on kasutatud uudset esitusviisi, mille kohaselt väliselektronid kirjelduvad tasalainetena.

\section{A. KUSHNIRENKO}

\section{A QUANTUM ELECTRODYNAMICAL MODEL OF NORMAL METAL}

An attempt is made to build a quantum electro-dynamical model of normal metal. In this model the normal metal is considered as the system of the electron and «lattice» (nuclear) fields interacting with an electromagnetic field according to Yukawa law. The morel is based on the relation between energy level of the system under consideration and the scattering matrix.

In this work all one-electron states are divided into internal (basic) and external (collective) ones. In the new representation the external electrons are described by plane waves, whereas the internal electrons are described by linear combinations of the atomic functions orthogonal to plane waves. To determine parameters entering into one-electron functions, a variation method is used.

The proposed model has the following advantages:

1) Since the external electrons are described in the new representation bv the plane waves all Coulomb and exchange integrals are calculated in an elementary way, which minimizes the computations.

2) The energy electron spectrum of the solid is defined in a closed analytical form convinient for calculating the physical properties of the solid.

3) The proposed model can be easily generalized for the disordered systems.

4) The correct calculation of the correlation energy of the external electrons goes beyond the limitations of the Chartry-Fock approximation. 